\title{
Studies towards C-3 functionalization of $\beta$-lactams using substituted allylsilanes
}

\author{
RENU THAPAR ${ }^{\mathrm{a}, *}$, RESHMA $^{\mathrm{b}}$ and S S BARI ${ }^{\mathrm{c}}$ \\ ${ }^{a} U$ I ET, Panjab University, Chandigarh 160014, India \\ ${ }^{b}$ D A V College, Sector 10, Chandigarh 160010, India \\ ${ }^{\mathrm{c}}$ Department of Chemistry and Centre of Advanced Studies in Chemistry, Panjab University, Chandigarh \\ 160014, India \\ e-mail: renuarorachem_pu@yahoo.co.in
}

MS received 17 April 2016; revised 1 August 2016; accepted 17 August 2016

\begin{abstract}
An effective and stereoselective synthesis of 3-(1'-methyl/phenylallyl)-3-phenylthio- $\beta$-lactams (3/4) using substituted allylsilane and Lewis acid is described. The reaction leads to the formation of a mixture of C-3 substituted allyl $\beta$-Lactams. However, these compounds on desulphurisation using tri-n-butyltinhydride and Raney Ni provide two separable diastereomers of the reduced product.
\end{abstract}

Keywords. Allylsilane; $\beta$-Lactams; Lewis acid; desulphurisation; crotylsilane; cinnamylsilane.

\section{Introduction}

The presence of $\beta$-lactam moiety in several widely used families of antibiotics has stimulated considerable activity directed at their stereocontrolled synthesis. ${ }^{1}$ In recent years, various natural monocyclic $\beta$-lactams have been shown to exhibit high antibacterial activity, suggesting that a suitably substituted monocyclic $\beta$-lactam might perhaps be the minimum requirement for biological activity. Besides, the ever growing new applications of $\beta$-lactam in various fields ranging from enzyme inhibition ${ }^{2}$ to the use of these products as starting materials to develop new synthetic methodology, ${ }^{3,4}$ justify a renewed interest in these compounds. ${ }^{5,6}$ In the $\beta$-lactam chemistry, introduction of allyl group at C-3 is of special interest. ${ }^{7}$ As olefins are considered as masked ketones, ${ }^{8}$ so the allylated $\beta$-lactams can be convenient intermediates for a variety of heterocyclic compounds. A limited number of approaches to densely functionalized $\alpha$-allyl- $\beta$-lactams have been reported so far. ${ }^{9}$ These employ appropriate substrate having carbonyl functionality at C-3 for their reactions with organometallics in organic as well as aqueous media. ${ }^{10}$ Alternate methods to introduce allyl group at $\beta$-lactam are of immense interest even today. As a part of our ongoing research and in continuation of earlier work, ${ }^{11}$ here we report a methodology capable of providing 3 -substituted allyl/propyl $\beta$-lactams. This

\footnotetext{
*For correspondence
}

employs the Lewis acid promoted addition of substituted allylsilane to $\beta$-lactam carbocation equivalents of type 2.

\section{Experimental}

\subsection{General Information}

Melting points (uncorrected) were determined with a Thomas-Hoover capillary melting point apparatus. Infrared spectra (IR) were recorded using Perkin-Elmer Model 1430 spectrophotometer. Only principal absorption bands of interest are reported in $\mathrm{cm}^{-1}$. ${ }^{1} \mathrm{H}$ NMR spectra were recorded using JEOL FT NMR System AL $300(300 \mathrm{MHz})$ in the Department of Chemistry and Centre of Advance Studies in Chemistry and BRUKER AVANCE II 400 (400 MHz) in the Sophisticated Analytical Instrumentation Facility (SAIF), Panjab University, Chandigarh. The chemical shift values are expressed in $\delta$ values ( $\mathrm{ppm})$ using tetramethylsilane (TMS) as internal standard. The coupling constant $J$ values are given in Hertz $(\mathrm{Hz})$. While reporting ${ }^{1} \mathrm{H}$ NMR data, following abbreviations have been used: s-singlet; br s-broad singlet; d-doublet; t-triplet; qquartet and m-multiplet. Thin layer chromatography (TLC) was performed using TLC grade silica gel ' $G$ ' (Acme Synthetic Chemicals). The spots were made visible by exposing plates to iodine vapor. Column chromatography was performed with silica gel (Acme Synthetic Chemicals, 60-120 mesh) and eluted with ethyl acetate:hexanes mixture unless otherwise stated. All commercially available compounds or reagents 
were used without further purification. All solvents used were of LR grade. Where necessary, the solvents were distilled and dried prior to use, when this seemed necessary by standard methods.

\subsection{General procedure for the synthesis of} cis-3-( $I^{\prime}$-methyl/phenylallyl)-3-phenylthio- $\beta$-lactams (3 and 4$)$

To a stirred solution of trans-3-chloro-3-phenylthio- $\beta$ lactam $2(1 \mathrm{mmol})$ and crotyl/ cinnamylsilane $(2 \mathrm{mmol})$ in dry methylene chloride $(15 \mathrm{~mL})$ at $0^{\circ} \mathrm{C}$ was added $\mathrm{TiCl}_{4}(2 \mathrm{mmol})$ rapidly under a nitrogen atmosphere. The resulting solution was stirred for additional $2 \mathrm{~h}$ at the same temperature. The reaction mixture was quenched with water, extracted with methylene chloride, washed with $5 \% \mathrm{NaHCO}_{3}$ solution, dried over $\mathrm{Na}_{2} \mathrm{SO}_{4}$ and filtered. The residue after solvent evaporation was purified by column chromatography.

2.2a 1-(4'-Methoxyphenyl)-3-(1'-methylallyl)-3-phenylthio-4-phenylazetidin-2-one (3a): Oil; yield 96\%; IR $v_{\max }\left(\mathrm{CHCl}_{3}\right): 1752(\mathrm{C}=\mathrm{O}), 1636.7,1512.8 \mathrm{~cm}^{-1} ;{ }^{1} \mathrm{H}$ NMR $\left(400 \mathrm{MHz}, \mathrm{CDCl}_{3}\right): \delta 6.74-7.65(\mathrm{~m}, 28 \mathrm{H}, \mathrm{Ph}$, both isomers), 6.24-6.39 (m, $1 \mathrm{H}$, minor isomer), 5.95$6.05(\mathrm{~m}, 1 \mathrm{H}$, major isomer), 5.15-5.30 $(\mathrm{m}, 4 \mathrm{H}$, both isomers), $5.00(\mathrm{~s}, 1 \mathrm{H}, \mathrm{C} 4-\mathrm{H}$, major isomer $), 4.97(\mathrm{~s}, 1 \mathrm{H}$, minor isomer), $3.75\left(\mathrm{~s}, 6 \mathrm{H}, \mathrm{OCH}_{3}\right.$, both isomers), 2.65-2.80 (m, 2H, both isomers), 1.40 (d, $J=8.8 \mathrm{~Hz}$, major isomer, $3 \mathrm{H}), 1.25(\mathrm{~d}, J=9.6 \mathrm{~Hz}$, minor isomer, $3 \mathrm{H}) ;{ }^{13} \mathrm{C}$ NMR $\left(100 \mathrm{MHz}, \mathrm{CDCl}_{3}\right): \delta 165.8,165.5$, $156.3,156.2,138.4,137.8,135.4,135.2,133.8,133.7$, $130.9,127.8,118.6,117.8,116.0,114.5,114.4,70.5$, 69.9, 60.7, 60.2, 55.2, 38.3, 37.3, 14.3, 13.8. Analysis: calcd. for $\mathrm{C}_{26} \mathrm{H}_{25} \mathrm{O}_{2} \mathrm{NS}$ : C, 75.15; H, 6.06; N, 3.37\%; Found: C, 75.27; H, 6.17; N, 3.42\%.

2.2b 1-(4'-Methylphenyl)-3-(1'-methylallyl)-3-phenylthio-4-(4'-chlorophenyl)azetidin-2-one (3b): Oil; yield $65 \%$; IR $v_{\max }\left(\mathrm{CCl}_{4}\right): 1757.1 \quad(\mathrm{C}=\mathrm{O}), \quad 1637.2$, $1615.2 \mathrm{~cm}^{-1} ;{ }^{1} \mathrm{H}$ NMR $\left(400 \mathrm{MHz}, \mathrm{CDCl}_{3}\right) \delta 7.00-7.64$ ( $\mathrm{m}, 28 \mathrm{H}, \mathrm{Ph}$, both isomers), 6.29-6.40 (m, 1H, minor isomer), 5.95-6.07 (m, 1H, major isomer), 5.13-5.33 $(\mathrm{m}, 4 \mathrm{H}$, both isomers), 4.99 (s, $1 \mathrm{H}, \mathrm{C} 4-\mathrm{H}$, major isomer), 4.96 (s, 1H, C4-H, minor isomer), 2.61-2.81 (m, $2 \mathrm{H}$, both isomers), 2.34 (s, $6 \mathrm{H}, \mathrm{CH}_{3}$, both isomers), $1.35\left(\mathrm{~d}, J=8.8 \mathrm{~Hz}, \mathrm{CH}_{3}\right.$, major isomer, $\left.3 \mathrm{H}\right), 1.23(\mathrm{~d}$, $J=9.5 \mathrm{~Hz}, \mathrm{CH}_{3}$, minor isomer, $\left.3 \mathrm{H}\right) ;{ }^{13} \mathrm{C} \mathrm{NMR}(100$ $\left.\mathrm{MHz}, \mathrm{CDCl}_{3}\right): \delta 165.8,165.5,138.1,137.6,135.9$, 135.2, 135.1, 135.0, 134.6, 134.5, 133.5, 133.4, 132.4, $132.2,130.5,130.4,129.8,129.7,129.6,129.5,129.3$, $129.2,129.1,129.0,128.9,128.8,128.5,128.4,118.0$, $117.5,117.3,116.2,70.6,69.8,60.1,59.1,38.5,37.2$,
21.2, 14.1, 13.8. Analysis: calcd. for $\mathrm{C}_{26} \mathrm{H}_{24} \mathrm{ONSCl}$ C, 72.03; H, 5.58; N, 3.23\%; Found: C, 72.17; H, 5.67; N, $3.34 \%$.

2.2c 1-(4'-Bromophenyl)-3-(1'-methylallyl)-3-phenylthio-4-phenylazetidin-2-one ( $3 c$ ): Oil; yield $81 \%$; IR $v_{\max }\left(\mathrm{CCl}_{4}\right): 1756.9(\mathrm{C}=\mathrm{O}), 1637.5,1590.9 \mathrm{~cm}^{-1} ;{ }^{1} \mathrm{H}$ NMR $\left(400 \mathrm{MHz}, \mathrm{CDCl}_{3}\right): \delta 7.14-7.63(\mathrm{~m}, 28 \mathrm{H}, \mathrm{Ph}$, both isomers), 6.29-6.40 (m, 1H, for minor isomer), 5.95-6.07 (m, 1H, major isomer), 5.12-5.22 (m, 4H, both isomers), 5.01 (s, 1H, C4-H, major isomer), 4.98 (s, $1 \mathrm{H}$, minor isomer), 2.64-2.78 (m, $2 \mathrm{H}$, both isomers), 1.35 (d, $J=8.7 \mathrm{~Hz}, 3 \mathrm{H}, \mathrm{CH}_{3}$, major isomer), $1.28\left(\mathrm{~d}, J=9.6 \mathrm{~Hz}, 3 \mathrm{H}, \mathrm{CH}_{3}\right.$, minor isomer $) ;{ }^{13} \mathrm{C}$ NMR (100 MHz, $\left.\mathrm{CDCl}_{3}\right): \delta 166.4,166.1,138.0,137.4$, 136.2, 136.1, 135.5, 135.4, 135.3, 133.2, 133.0, 132.3, $132.2,130.5,130.4,128.8,128.5,128.4,128.2,128.1$, $128.0,118.9,118.3,117.0,116.9,116.3,70.8,70.1$, 60.1, 60.3, 38.2, 37.1, 14.2, 13.7. Analysis: calcd. for $\mathrm{C}_{25} \mathrm{H}_{22} \mathrm{ONSBr}$ : C, 64.78; H, 4.79; N, 3.02\%; Found: C, $64.87 ; \mathrm{H}, 4.83 ; \mathrm{N}, 3.10 \%$.

2.2d 1-Phenyl-3-(1'-methylallyl)-3-phenylthio-4-phenylazetidin-2-one (3d): Oil; yield 85\%; IR $v_{\max }\left(\mathrm{CHCl}_{3}\right)$ : $1757.4(\mathrm{C}=\mathrm{O}), 1600.7 \mathrm{~cm}^{-1}$; ${ }^{1} \mathrm{H}$ NMR $(400 \mathrm{MHz}$, $\left.\mathrm{CDCl}_{3}\right): \delta 7.01-7.64(\mathrm{~m}, 20 \mathrm{H}, \mathrm{Ph}$ both isomers), 6.30 $6.42(\mathrm{~m}, 1 \mathrm{H}$, minor isomer), 5.95-6.05 $(\mathrm{m}, 1 \mathrm{H}$, major isomer), $5.16-5.31(\mathrm{~m}, 4 \mathrm{H}$, both isomers), $5.04(\mathrm{~s}, 1 \mathrm{H}$, $\mathrm{C} 4-\mathrm{H}$, major isomer), 5.01 ( $\mathrm{s}, 1 \mathrm{H}, \mathrm{C} 4-\mathrm{H}$, minor isomer), 2.66-2.81 (m, 2H, both isomers), 1.35 (d, $J=8.7 \mathrm{~Hz}$, $3 \mathrm{H}, \mathrm{CH}_{3}$, major isomer), $1.25\left(\mathrm{~d}, J=9.6 \mathrm{~Hz}, 3 \mathrm{H}, \mathrm{CH}_{3}\right.$, minor isomer); $\left.{ }^{13} \mathrm{C} \mathrm{NMR} \mathrm{(100} \mathrm{MHz,} \mathrm{CDCl}_{3}\right): \delta 166.3$, 166.0, 138.2, 137.6, 137.3, 137.2, 135.4, 135.3, 133.6, $133.5,130.7,130.6,129.2,129.1,128.7,128.6,128.5$, $128.4,128.3,128.2,128.1,128.0,127.9,124.0,123.9$, 117.9, 116.0, 70.5, 69.8, 60.6, 60.1, 38.4, 37.2, 14.2, 13.9. Analysis: calcd. for $\mathrm{C}_{25} \mathrm{H}_{23}$ ONS: C, 77.89; H, 6.01; N, 3.63\%; Found: C, 77.97; H, 6.10; N, 3.70\%.

2.2e 1-(4'-Methoxyphenyl)-3-(1'-phenylallyl)-3-phenylthio-4-phenylazetidin-2-one (4a): Oil; yield 62\%; IR $v_{\max }\left(\mathrm{CCl}_{4}\right): 1754(\mathrm{C}=\mathrm{O}) \mathrm{cm}^{-1} ;{ }^{1} \mathrm{H}$ NMR $(400 \mathrm{MHz}$, $\left.\mathrm{CDCl}_{3}\right): \delta 6.95-7.58(\mathrm{~m}, 38 \mathrm{H}, \mathrm{Ph}$, both isomers), 6.50$6.70(\mathrm{~m}, 2 \mathrm{H}$, both isomers), 5.20-5.57 (m, 6H, both isomers), 4.15-4.20 ( $\mathrm{m}, 2 \mathrm{H}$, both isomers), 3.98 (s, $3 \mathrm{H}, \mathrm{OCH}_{3}$, major isomer), $3.94\left(\mathrm{~s}, 3 \mathrm{H}, \mathrm{OCH}_{3}\right.$, minor isomer); $\left.{ }^{13} \mathrm{C} \mathrm{NMR} \mathrm{(100} \mathrm{MHz,} \mathrm{CDCl}_{3}\right): \delta 165.2,164.8$, 156.2, 156.0, 138.8, 138.4, 136.2, 135.5, 135.4, 135.1, $133.8,130.8,130.5,129.7,128.6,128.5,128.4,128.3$, 128.2, 128.1, 128.0, 118.9, 118.6, 118.4, 114.5, 114.3, 70.5, 70.1, 61.8, 61.4, 55.1, 55.0, 51.7, 51.2. Analysis: calcd. for $\mathrm{C}_{31} \mathrm{H}_{27} \mathrm{O}_{2} \mathrm{NS}$ : C, 77.95; H, 5.69; N, 2.93\%; Found: C, 78.08; H, 5.77; N, 2.99\%. 


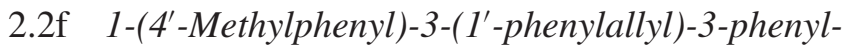
thio-4-(4'-chlorophenyl)azetidin-2one $(\mathbf{4 b})$ : Oil; yield $71 \%$; IR $v_{\max }\left(\mathrm{CCl}_{4}\right): 1761(\mathrm{C}=\mathrm{O}) \mathrm{cm}^{-1} ;{ }^{1} \mathrm{H}$ NMR $\left(400 \mathrm{MHz}, \mathrm{CDCl}_{3}\right): \delta$ 7.45-8.00 $(\mathrm{m}, 36 \mathrm{H}, \mathrm{Ph}$, both isomers), 6.56-6.75 (m, 2H, both isomers), 5.45 (s, $2 \mathrm{H}$, $\mathrm{C} 4-\mathrm{H}$, both isomers), 5.23-5.66 ( $\mathrm{m}, 4 \mathrm{H}$, both isomers), 4.17-4.22 (m, $2 \mathrm{H}$, both isomers), $2.54\left(\mathrm{~s}, 3 \mathrm{H}, \mathrm{CH}_{3}\right.$, major isomer), 2.43 (s, $3 \mathrm{H}, \mathrm{CH}_{3}$, minor isomer); ${ }^{13} \mathrm{C}$ NMR (100 MHz, $\left.\mathrm{CDCl}_{3}\right) \delta$ 165.2, 164.8, 138.6, 138.2, 136.4, 135.5, 135.2, 135.1, 133.6, 130.7, 130.2, 129.5, 128.6, 128.5, 128.4, 128.3, 128.2, 128.1, 128.0, 118.7, 118.5, 118.4, 114.4, 114.1, 70.3, 70.1, 61.9, 61.5, 51.5, 51.3, 21, 20.9. Analysis: calcd. for $\mathrm{C}_{31} \mathrm{H}_{26} \mathrm{ONSCl}$ : C, 75.13; H, 5.29; N, 2.83\%; Found: C, 75.18; H, 5.37; $\mathrm{N}, 2.90 \%$.

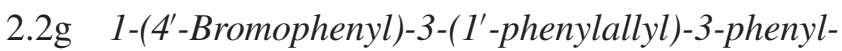
thio-4-phenylazetidin-2-one (4c): Oil; yield 71\%; IR $v_{\max }\left(\mathrm{CCl}_{4}\right): 1760(\mathrm{C}=\mathrm{O}), 1656 \mathrm{~cm}^{-1} ;{ }^{1} \mathrm{H}$ NMR $\left(400 \mathrm{MHz}, \mathrm{CDCl}_{3}\right): \delta 7.00-7.98(\mathrm{~m}, 38 \mathrm{H}, \mathrm{Ph}$, both isomers), $6.52-6.72(\mathrm{~m}, 2 \mathrm{H}$, both isomers), $5.40(\mathrm{~s}, 2 \mathrm{H}$, $\mathrm{C} 4-\mathrm{H}$, both isomers), $5.20-5.60$ ( $\mathrm{m}, 4 \mathrm{H}$, both isomers), 4.17-4.22 (m, 2H, both isomers); ${ }^{13} \mathrm{C}$ NMR (100 $\left.\mathrm{MHz}, \mathrm{CDCl}_{3}\right): \delta 166.4,166.1,139.1,138.7,136.5$, 136.4, 136.3, 136.1, 135.8, 135.7, 135.6, 133.8, 133.0, $132.9,132.7,132.5,131.1,131.0,130.6,130.3,132.9$, $132.7,132.5,131.1,131.0,130.6,130.3,130.2,130.0$, $129.8,129.5,129.3,129.1,129.0,128.9,128.8,128.7$, 128.6, 128.5, 128.3, 128.2, 127.9, 127.7, 120.6, 119.8, $119.7,119.5,119.4,119.2,118.4,117.6,117.4,71.5$, 70.9, 69.4, 62.6, 62.0, 52.4, 51.6. Analysis: calcd. for $\mathrm{C}_{30} \mathrm{H}_{24} \mathrm{ONSBr}$ : C, 68.56; H, 4.60; N, 2.66\%; Found: C, $68.59 ; \mathrm{H}, 4.67 ; \mathrm{N}, 2.73 \%$.

2.2h 1-Phenyl-3-(1'-phenylallyl)-3-phenylthio-4phenylazetidin-2-one $(\mathbf{4 d})$ : Oil; yield 70\%; IR $v_{\max }$ $\left(\mathrm{CCl}_{4}\right): 1755(\mathrm{C}=\mathrm{O}) \mathrm{cm}^{-1} ;{ }^{1} \mathrm{H}$ NMR $(400 \mathrm{MHz}$, $\left.\mathrm{CDCl}_{3}\right): \delta 7.00-7.90(\mathrm{~m}, 40 \mathrm{H}, \mathrm{Ph}$, both isomers), $6.60(\mathrm{~m}, 2 \mathrm{H}$, both isomers), $5.49(\mathrm{~s}, 2 \mathrm{H}, \mathrm{C} 4-\mathrm{H}$, both isomers), $5.18-5.68(\mathrm{~m}, 4 \mathrm{H}$, both isomers $), 4.16(\mathrm{~m}$, $2 \mathrm{H}$, both isomers); ${ }^{13} \mathrm{C} \mathrm{NMR}\left(100 \mathrm{MHz}, \mathrm{CDCl}_{3}\right): \delta$ $166.8,166.4,160.8,140.0,138.9,137.2,136.5,135.9$, $135.7,134.3,138.7,131.5,131.3,131.2,130.5,130.3$, $122.5,121.9,121.5,121.4,117.5,117.1,80.7,71.2$, $70.8,69.5,62.5,62.2,52.3,52.0$. Analysis: calcd. for $\mathrm{C}_{30} \mathrm{H}_{25}$ ONS: C, 80.50; H, 5.63; N, 3.13\%; Found: C, 80.55; H, 5.69; N, 3.23\%.

\subsection{General procedure for $n-B u_{3} S n H$ reduction}

To a solution of $\mathbf{3 / 4}(1 \mathrm{mmol})$ in dry toluene $(10 \mathrm{~mL})$ were added $n-\mathrm{Bu}_{3} \mathrm{SnH}(1 \mathrm{mmol})$ and catalytic amount of AIBN. The reaction mixture was refluxed and progress of the reaction was monitored by TLC. After completion of reaction, the reaction mixture was cooled to RT and concentrated under vacuum. The residue was redissolved in DCM $(20 \mathrm{~mL})$ and washed with water. The residue obtained after evaporation of solvent was purified by silica gel column chromatography.

2.3a cis-1-(4'-Methoxyphenyl)-3-(1'-methylallyl)-4phenylazetidin-2-one $(\mathbf{5 a}, \mathbf{6} \boldsymbol{a})$ : The fast moving diastereomer 5a was characterized by the following data: White solid; yield 24\%; M.p. $125-127^{\circ} \mathrm{C}$; IR $v_{\max }$ (KBr): $1749(\mathrm{C}=\mathrm{O}), 1513 \mathrm{~cm}^{-1}$; ${ }^{1} \mathrm{H}$ NMR $(400 \mathrm{MHz}$, $\left.\mathrm{CDCl}_{3}\right): \delta 7.08-7.29(\mathrm{~m}, 9 \mathrm{H}, \mathrm{Ph}), 5.11-5.25(\mathrm{~m}, 1 \mathrm{H})$, $5.05(\mathrm{~d}, J=7.6 \mathrm{~Hz}, 1 \mathrm{H}), 4.58(\mathrm{~d}, J=12 \mathrm{~Hz} 1 \mathrm{H}), 4.25$ $(\mathrm{d}, J=17 \mathrm{~Hz}, 1 \mathrm{H}), 3.69\left(\mathrm{~s}, 3 \mathrm{H}, \mathrm{OCH}_{3}\right), 3.28-3.35(\mathrm{~m}$, $1 \mathrm{H}), 2.23-2.30(\mathrm{~m}, 1 \mathrm{H}), 1.23(\mathrm{~d}, J=6 \mathrm{~Hz} 3 \mathrm{H}) ;{ }^{13} \mathrm{C}$ NMR (100 MHz, $\left.\mathrm{CDCl}_{3}\right): \delta 165.5,155.8,140.5,135.4$, $131.4,128.5,128.4,123.2,118.3,114.3,113.9,59.2$, 58.7, 55.1, 34.7, 18.4. Analysis: calcd. for $\mathrm{C}_{20} \mathrm{H}_{21} \mathrm{O}_{2} \mathrm{~N}$ : C, 78.14; H, 6.88; N, 4.56\%; Found: C, 78.27; H, 6.99; $\mathrm{N}, 4.69 \%$.

The slow moving diastereomer $\mathbf{6 a}$ was characterized by following data: Solid; yield $54 \%$; M.p. $105-107^{\circ} \mathrm{C}$; IR $v_{\max }(\mathrm{KBr}): 1749(\mathrm{C}=\mathrm{O}), 1513.3 \mathrm{~cm}^{-1} ;{ }^{1} \mathrm{H}$ NMR $\left(400 \mathrm{MHz}, \mathrm{CDCl}_{3}\right): \delta$ 7.11-7.36 (m, 9H, Ph), 5.95-6.16 $(\mathrm{m}, 1 \mathrm{H}), 5.07(\mathrm{~d}, J=8 \mathrm{~Hz}, 1 \mathrm{H}), 4.89-5.02(\mathrm{~m}, 2 \mathrm{H})$, $3.72\left(\mathrm{~s}, 3 \mathrm{H}, \mathrm{OCH}_{3}\right), 3.28-3.35(\mathrm{~m}, 1 \mathrm{H}), 2.20-2.26(\mathrm{~m}$, $1 \mathrm{H}), 0.6(\mathrm{~d}, J=4.4 \mathrm{~Hz}, 3 \mathrm{H}) ;{ }^{13} \mathrm{C} \mathrm{NMR}(100 \mathrm{MHz}$, $\left.\mathrm{CDCl}_{3}\right): \delta 165.0,155.9,140.2,135.5,131.4,128.7$, $128.5,128.3,127.9,127.5,126.9,118.3,114.4,114.3$, $60.6,58.5,55.1,34.3,17.6$.

2.3b cis-1-Phenyl-3-(1'-methylallyl)-4-phenylazetidin2-one $(\mathbf{5 d}, \boldsymbol{6} \boldsymbol{d})$ : The fast moving diastereomer $\mathbf{5 d}$ was characterized by following data: White solid; yield 28\%; M.p. 86-88 ${ }^{\circ}$; IR $v_{\max }(\mathrm{KBr}): 1750(\mathrm{C}=\mathrm{O})$, $1512 \mathrm{~cm}^{-1} ;{ }^{1} \mathrm{H}$ NMR $\left(400 \mathrm{MHz}, \mathrm{CDCl}_{3}\right): \delta 6.86-7.24$ $(\mathrm{m}, 10 \mathrm{H}, \mathrm{Ph}), 5.05-5.15(\mathrm{~m}, 1 \mathrm{H}), 5.03(\mathrm{~d}, J=7.6$ $\mathrm{Hz}, 1 \mathrm{H}), 4.54(\mathrm{~d}, J=12 \mathrm{~Hz}, 1 \mathrm{H}), 4.20(\mathrm{~d}, J=17.2$ $\mathrm{Hz}, 1 \mathrm{H}), 3.26-3.32(\mathrm{~m}, 1 \mathrm{H}), 2.22-2.27(\mathrm{~m}, 1 \mathrm{H}), 1.17$ $(\mathrm{d}, J=6 \mathrm{~Hz}), 3 \mathrm{H}) ;{ }^{13} \mathrm{C}$ NMR $\left(100 \mathrm{MHz}, \mathrm{CDCl}_{3}\right): \delta$ 166.1, 140.8, 140.4, 139.8, 137.7, 135.1, 129.2, 129.1, 129.0, 128.4, 128.3, 123.2, 128.1, 126.1, 126.0, 123.4, 117.1, 116.9, 115.5, 113.9, 65.1, 59.1, 58.6, 34.6, 18.4. Analysis: calcd. for $\mathrm{C}_{19} \mathrm{H}_{19} \mathrm{ON}$ : C, 82.26; H, 6.90; N, 5.05\%; Found: C, 82.32; H, 6.97; N, 5.11\%.

The slow moving diastereomer $\mathbf{6 d}$ was characterized by following data: White solid; yield 56\%; M.p. 126$128^{\circ} \mathrm{C}$; IR $v_{\max }(\mathrm{KBr}): 1750(\mathrm{C}=\mathrm{O}), 1514 \mathrm{~cm}^{-1} ;{ }^{1} \mathrm{H}$ NMR (400 MHz, $\left.\mathrm{CDCl}_{3}\right): \delta 6.87-7.30(\mathrm{~m}, 9 \mathrm{H}, \mathrm{Ph})$, 5.93-6.06 (m, 1H), $5.10(\mathrm{~d}, J=8 \mathrm{~Hz}, 1 \mathrm{H}), 4.84-5.07$ (m, 2H), 3.26-3.33 (m, 1H), 2.23-2.28 (m, 1H), 0.56 
$(\mathrm{d}, J=4.4 \mathrm{~Hz}, 3 \mathrm{H}) ;{ }^{13} \mathrm{C} \mathrm{NMR}\left(100 \mathrm{MHz}, \mathrm{CDCl}_{3}\right): \delta$ 165.70, 140.1, 137.8, 135.2, 129.0, 128.7, 128.6, 127.8, $123.6,117.1,114.4,60.5,58.4,34.3,17.6$.

2.3c cis-1-(4'-Methoxyphenyl)-3-(1'-phenylallyl)-4phenylazetidin-2-one $(7 a, 8 a)$ : The fast moving diastereomer $7 \mathbf{a}$ was characterized by following data: Solid; yield 49\%; M.p. $112-114^{\circ} \mathrm{C}$; IR $v_{\max }(\mathrm{KBr})$ : $1745(\mathrm{C}=\mathrm{O}), 1515 \mathrm{~cm}^{-1} ;{ }^{1} \mathrm{H}$ NMR $\left(400 \mathrm{MHz}, \mathrm{CDCl}_{3}\right)$ : $\delta$ 6.53-7.29 (m, 14H, Ph), 6.25-6.27 (m, 1H), $5.05(\mathrm{~d}$, $J=5 \mathrm{~Hz}, 1 \mathrm{H}), 4.98(\mathrm{~d}, J=5.4 \mathrm{~Hz}, 1 \mathrm{H}), 4.62-4.68$ $(\mathrm{m}, 1 \mathrm{H}), 4.04-4.10(\mathrm{~m}, 1 \mathrm{H}), 3.70(\mathrm{~s}, 3 \mathrm{H}), 3.36-3.40$ $(\mathrm{m}, 1 \mathrm{H}),{ }^{13} \mathrm{C}$ NMR $\left(100 \mathrm{MHz}, \mathrm{CDCl}_{3}\right): \delta 165,156.1$, $137.8,137.4,136,135.5,135.4,135.1,133.5,130.4$, $130.2,129.5,128.6,128.2,128.7,128.3,128.2,128.1$, $128.0,118.8,118.6,118,114.5,114.2,61,60,55.1$, 50. Analysis: calcd for $\mathrm{C}_{25} \mathrm{H}_{23} \mathrm{O}_{2} \mathrm{~N}$ : Calcd. C, 81.27; H, 6.27; N, 3.79\%; Found: C, 81.38; H, 6.39; N, 3.89\%.

The slow moving diastereomer $\mathbf{8 a}$ was characterized by following data: Solid; yield $25 \%$; M.p. $182-184^{\circ} \mathrm{C}$; IR $v_{\max }(\mathrm{KBr}): 1749(\mathrm{C}=\mathrm{O}), 1512 \mathrm{~cm}^{-1} ;{ }^{1} \mathrm{H}$ NMR $(400$ $\left.\mathrm{MHz}, \mathrm{CDCl}_{3}\right): \delta 6.66-7.27(\mathrm{~m}, 14 \mathrm{H}, \mathrm{Ph}), 5.47-5.59(\mathrm{~m}$, $1 \mathrm{H}), 5.18(\mathrm{~d}, J=8 \mathrm{~Hz}, 1 \mathrm{H}), 4.74-4.78(\mathrm{~m}, 1 \mathrm{H}), 4.46-$ $4.52(\mathrm{~m}, 1 \mathrm{H}), 3.96-4.02(\mathrm{~m}, 1 \mathrm{H}), 3.69(\mathrm{~s}, 3 \mathrm{H}), 3.36-$ $3.38(\mathrm{~m}, 1 \mathrm{H}) ;{ }^{13} \mathrm{C}$ NMR $\left(100 \mathrm{MHz}, \mathrm{CDCl}_{3}\right): \delta 165.2$, 156.0, 138.8, 138.4, 136.2, 135.5, 135.4, 135.1, 133.8, $130.8,130.5,129.7,128.6,128.5,128.4,128.3,128.2$, $128.1,128.0,118.9,118.6,118.4,114.5,114.3,61.2$, $60.5,55.0,51.5$.

2.3d cis-1-Phenyl-3-(1'-phenylallyl)-4-phenylazetidin2-one $(\mathbf{7 d}, \boldsymbol{8 d})$ : The fast moving diastereomer $\mathbf{7 d}$ was characterized by following data: Oil; yield 44\%; IR $v_{\max }\left(\mathrm{CCl}_{4}\right): 1750(\mathrm{C}=\mathrm{O}), 1512 \mathrm{~cm}^{-1} ;{ }^{1} \mathrm{H}$ NMR (400 $\left.\mathrm{MHz}, \mathrm{CDCl}_{3}\right): \delta 6.97-7.30(\mathrm{~m}, 15 \mathrm{H}, \mathrm{Ph}), 6.27-6.29$ $(\mathrm{m}, 1 \mathrm{H}), 5.03(\mathrm{~d}, J=5.4 \mathrm{~Hz}, 1 \mathrm{H}), 4.96(\mathrm{~d}, J=5 \mathrm{~Hz}$, $1 \mathrm{H}), 4.65-4.70(\mathrm{~m}, 1 \mathrm{H}), 4.04-4.10(\mathrm{~m}, 1 \mathrm{H}), 3.35-3.40$ $(\mathrm{m}, 1 \mathrm{H}) ;{ }^{13} \mathrm{C}$ NMR $\left(100 \mathrm{MHz}, \mathrm{CDCl}_{3}\right): \delta 166.8,139.0$, 138.6, 137.1, 136.5, 135.8, 135.7, 134.1, 132.7, 131.5, 131.1, 131.0, 130.5, 130.2, 122.2, 121.7, 121.6, 121.3, 117.3, 117.1, 61.7, 60.5, 51.0. Analysis: calcd. for $\mathrm{C}_{24} \mathrm{H}_{21} \mathrm{ON}$ : Calcd C, 84.91; H, 6.24; N, 4.13\%; Found: C, 84.97; H, 6.30; N, 4.19\%.

The slow moving diastereomer $\mathbf{8 d}$ was characterized by following data: Solid; yield $29 \%$; M.p. $153-160^{\circ} \mathrm{C}$; IR $v_{\max }(\mathrm{KBr}): 1752(\mathrm{C}=\mathrm{O}), 1513 \mathrm{~cm}^{-1} ;{ }^{1} \mathrm{H}$ NMR $(400$ $\left.\mathrm{MHz}, \mathrm{CDCl}_{3}\right): \delta 6.45-7.32(\mathrm{~m}, 10 \mathrm{H}, \mathrm{Ph}), 5.43-5.52(\mathrm{~m}$, $1 \mathrm{H}), 5.22(\mathrm{~d}, J=8 \mathrm{~Hz}, 1 \mathrm{H}), 4.73-4.77(\mathrm{~m}, 1 \mathrm{H}), 4.45-$ $4.51(\mathrm{~m}, 1 \mathrm{H}), 3.95-4.02(\mathrm{~m}, 1 \mathrm{H}), 3.35-3.37(\mathrm{~m}, 1 \mathrm{H})$; ${ }^{13} \mathrm{C}$ NMR $\left(100 \mathrm{MHz}, \mathrm{CDCl}_{3}\right): \delta 166.2,139.0,138.4$, $137,136.2,135.8,135.2,134.4,132.7,131.7,131.3$, 131.0, 130.8, 130.6, 122.4, 121.5, 121.3, 121.1, 117.2, 117, 61.9, 60.2, 51.3.

\subsection{General procedure for Raney-Ni reduction}

To a suspension of Raney-Ni ( $15 \mathrm{mmol}, 100 \%$ activated) in dry ethanol $(20 \mathrm{~mL})$ was added $\mathbf{3 / 4}(1 \mathrm{mmol})$. The suspension was refluxed for $1 \mathrm{~h}$. The progress of reaction was checked by TLC. After disappearance of spot for the starting substrate and appearance of a new spot, suspension was filtered and solvent was evaporated in vacuo, extracted with methylene chloride $(3 \times 20 \mathrm{~mL})$ and dried over anhydrous $\mathrm{Na}_{2} \mathrm{SO}_{4}$. The residue so obtained was purified by silica gel column chromatography.

2.4a cis-1-(4'-Methoxyphenyl)-3-(1'-methylpropyl)-4phenylazetidin-2-one $(\mathbf{9 a})$ : Oil; yield $100 \%$; IR $v_{\max }$ $\left(\mathrm{CCl}_{4}\right)$ : $1745.6(\mathrm{C}=\mathrm{O}) \mathrm{cm}^{-1} ;{ }^{1} \mathrm{H}$ NMR $(400 \mathrm{MHz}$, $\left.\mathrm{CDCl}_{3}\right): \delta 6.67-7.35(\mathrm{~m}, 9 \mathrm{H}, \mathrm{Ph}), 5.04(\mathrm{~d}, J=8 \mathrm{~Hz}$, $1 \mathrm{H}), 3.69(\mathrm{~s}, 3 \mathrm{H}), 3.00-3.20(\mathrm{~m}, 1 \mathrm{H}), 1.06(\mathrm{~d}, J=6$ $\mathrm{Hz}, 3 \mathrm{H}), 0.84$ (t, $J=10 \mathrm{~Hz}, 2 \mathrm{H}), 0.60-0.70(\mathrm{~m}, 1 \mathrm{H})$, $0.40-0.50(\mathrm{~m}, 3 \mathrm{H}) ;{ }^{13} \mathrm{C}$ NMR $\left(100 \mathrm{MHz}, \mathrm{CDCl}_{3}\right): \delta$ 166.1, 155.7, 135.6, 131.4, 129.1, 128.6, 128.3, 127.8, $127.2,125.9,118.2,117.7,114.2,60.8,60.1,58.6$, 55.1, 31.6, 31.5, 27.5, 26.7, 16.9, 16.7, 10.7. 10.4. Analysis: calcd. for $\mathrm{C}_{20} \mathrm{H}_{23} \mathrm{O}_{2} \mathrm{~N}$ : C, 77.63; H, 7.49; N, 4.52\%; Found: C, 77.77; H, 7.62; N, 4.61\%.

2.4b cis-1-Phenyl-3-(1'-methylpropyl)-4-phenylazetidin2-one (9d): Oil; yield 80\%; IR $v_{\max }\left(\mathrm{CCl}_{4}\right): 1745$ $(\mathrm{C}=\mathrm{O}) \mathrm{cm}^{-1} ;{ }^{1} \mathrm{H}$ NMR $\left(400 \mathrm{MHz}, \mathrm{CDCl}_{3}\right): \delta 6.80$ $7.25(\mathrm{~m}, 10 \mathrm{H}, \mathrm{Ph}), 5.00(\mathrm{~d}, J=8 \mathrm{~Hz}, 1 \mathrm{H}), 3.00-3.23$ $(\mathrm{m}, 1 \mathrm{H}), 1.00(\mathrm{~d}, J=6 \mathrm{~Hz}, 3 \mathrm{H}), 0.74(\mathrm{t}, J=10 \mathrm{~Hz}$, 2H), 0.60-0.71 (m, 1H), 0.41-0.50 (m, 3H); ${ }^{13} \mathrm{C}$ NMR $\left(100 \mathrm{MHz}, \mathrm{CDCl}_{3}\right): \delta 166,135.4,131.2,129.4,128.5$, $128.2,127.5,127.2,125.7,118.2,117.6,114.5,60.6$, 60.2, 58.4, 55, 31.7, 31.5, 27.4, 26.5, 16.6, 16.5, 10.8 . 10.5. Analysis: calcd. for $\mathrm{C}_{19} \mathrm{H}_{20} \mathrm{ON}$ : C, 81.96; H, 7.24; N, 5.03\%; Found: C, 81.99; H, 7.30; N, 5.13\%.

2.4c cis-1-(4'-Methoxyphenyl)-3-(1'-phenylpropyl)-4phenylazetidin-2-one $(\mathbf{1 0 a}, \mathbf{1 1 a})$ : The fast moving diastereomer 10a was characterized by following data: Oil; yield 32\%; IR $v_{\max }\left(\mathrm{CCl}_{4}\right)$ : $1745.3(\mathrm{C}=\mathrm{O}) \mathrm{cm}^{-1}$; ${ }^{1} \mathrm{H}$ NMR $\left(400 \mathrm{MHz}, \mathrm{CDCl}_{3}\right): \delta 6.35-7.21(\mathrm{~m}, 14 \mathrm{H}$, $\mathrm{Ph}), 4.83(\mathrm{~d}, J=7.6 \mathrm{~Hz}, 1 \mathrm{H}), 3.86(\mathrm{dd}, J=8,16 \mathrm{~Hz}$, $1 \mathrm{H}), 3.63(\mathrm{~s}, 3 \mathrm{H}), 2.40-2.49(\mathrm{~m}, 1 \mathrm{H}), 2.24-2.32(\mathrm{~m}$, 1H), $1.40-1.50(\mathrm{~m}, 1 \mathrm{H}), 0.55(\mathrm{t}, J=10 \mathrm{~Hz}, 3 \mathrm{H}) ;{ }^{13} \mathrm{C}$ NMR $\left(100 \mathrm{MHz}, \mathrm{CDCl}_{3}\right): \delta 165.9,155.8,141.5,135.0$, $131.3,129.1,128.7,128.4,128.2,128.1,127.8,127.7$, $127.5,127.0,125.9,118.3,118.1,114.3,114.2,65.9$, 59.3, 58.3, 55.1, 47.1, 44.4, 27.9, 26.0, 12.3, 11.3.

The slow moving diastereomer 11a was characterized by following data: Solid; yield 45\%; M.p. 187$189^{\circ} \mathrm{C}$; IR $v_{\max }(\mathrm{KBr}): 1746.1(\mathrm{C}=\mathrm{O}) \mathrm{cm}^{-1} ;{ }^{1} \mathrm{H}$ NMR 
$\left(400 \mathrm{MHz}, \mathrm{CDCl}_{3}\right): \delta 6.72-7.40(\mathrm{~m}, 14 \mathrm{H}, \mathrm{Ph}), 5.23$ $(\mathrm{d}, J=7.6 \mathrm{~Hz}, 1 \mathrm{H}), 3.82-3.88(\mathrm{~m}, 1 \mathrm{H}), 2.40-2.52(\mathrm{~m}$, $1 \mathrm{H}), 1.34-1.40(\mathrm{~m}, 2 \mathrm{H}), 0.39(\mathrm{t}, J=9.6 \mathrm{~Hz}, 3 \mathrm{H}) ;{ }^{13} \mathrm{C}$ NMR $\left(100 \mathrm{MHz}, \mathrm{CDCl}_{3}\right): \delta 165.5,155.7,141.3,135.0$, 131.3, 128.6, 128.3, 128.2, 127.6, 126.5, 118.2, 114.2, 60.0, 58.9, 55.3, 44.4, 27.8, 11.2. Analysis: calcd. for $\mathrm{C}_{25} \mathrm{H}_{25} \mathrm{O}_{2} \mathrm{~N}$ : C, 81.83; H, 6.78; N, 3.77\%; Found: C, $81.95 ; \mathrm{H}, 6.90 ; \mathrm{N}, 3.89 \%$.

2.4d cis-1-Phenyl-3-(1'-phenylpropyl)-4-phenylazetidin2-one $(10 \mathrm{~d}, 11 \mathrm{~d})$ : The fast moving diastereomer $10 \mathrm{~d}$ was characterized by following data: Oil; yield 39\%; IR $v_{\max }\left(\mathrm{CCl}_{4}\right): 1745.3(\mathrm{C}=\mathrm{O}) \mathrm{cm}^{-1} ;{ }^{1} \mathrm{H}$ NMR $(400$ $\left.\mathrm{MHz}, \mathrm{CDCl}_{3}\right): \delta 6.36-7.25(\mathrm{~m}, 15 \mathrm{H}, \mathrm{Ph}$, both isomers), $4.87(\mathrm{~d}, J=7.6 \mathrm{~Hz}, 1 \mathrm{H}), 3.86(\mathrm{dd}, J=8,16 \mathrm{~Hz}$, $1 \mathrm{H}), 2.41-2.50(\mathrm{~m}, 1 \mathrm{H}), 2.25-2.33(\mathrm{~m}, 1 \mathrm{H}), 1.41-1.51$ $(\mathrm{m}, 1 \mathrm{H}), 0.54(\mathrm{t}, J=10 \mathrm{~Hz}, 3 \mathrm{H}) ;{ }^{13} \mathrm{C}$ NMR $(100$ $\left.\mathrm{MHz}, \mathrm{CDCl}_{3}\right): \delta 166.5,141.5,137.7,134.9,129.2$, $129.0,128.9,128.7,128.4,128.3,127.9,127.8,127.5$, 127.1, 126.0, 125.9, 123.6, 117.2, 117.1, 116.9, 59.4, 58.3, 47.1, 44.4, 27.9, 27.0, 11.3. Analysis: calcd. for $\mathrm{C}_{24} \mathrm{H}_{23} \mathrm{ON}$ : C, 84.41; H, 6.79; N, 4.10\%; Found: C, 84.45; H, 6.84; N, 4.15\%.

The slow moving diastereomer 11d was characterized by following data: Solid; yield 64\%; M.p. 173$175^{\circ} \mathrm{C}$; IR $v_{\max }(\mathrm{KBr}): 1745(\mathrm{C}=\mathrm{O}) \mathrm{cm}^{-1} ;{ }^{1} \mathrm{H}$ NMR $\left(400 \mathrm{MHz}, \mathrm{CDCl}_{3}\right): \delta 6.90-7.37(\mathrm{~m}, 15 \mathrm{H}, \mathrm{Ph}), 5.21(\mathrm{~d}$, $J=7.6 \mathrm{~Hz}, 1 \mathrm{H}), 3.82-3.87(\mathrm{~m}, 1 \mathrm{H}), 2.42-2.53(\mathrm{~m}$, $1 \mathrm{H}), 1.35-1.42(\mathrm{~m}, 2 \mathrm{H}), 0.40(\mathrm{t}, J=9.6 \mathrm{~Hz}, 3 \mathrm{H}) ;{ }^{13} \mathrm{C}$ NMR $\left(100 \mathrm{MHz}, \mathrm{CDCl}_{3}\right): \delta 166.2,141.0,137.0,134.7$, $128.2,127.8,127.6,126.9,126.4,122.7,116.2,59.6$, $57.7,30.8,26.8,15.9,9.6$.

\section{Results and Discussion}

It has been previously reported that exposure of trans3 -chloro-3-phenylthio- $\beta$-lactams and allylsilane to a Lewis acid promotes a facile and stereoselective C-3 allylation to give cis-3-allyl-3-phenylthio- $\beta$-lactams in excellent yield. ${ }^{11-13}$ The present work is an extension of the same methodology employing the use of substituted silanes. The starting substrates for Lewis acid mediated C-3 functionalization, i.e., trans-3-chloro3 -phenylthio- $\beta$-lactams 2 were prepared by reacting phenylthioacetyl chloride or potassium phenylthioacetate with Schiff base in the presence of a triethylamine ${ }^{8}$ and phoshphorus oxychloride, followed by stereospecific chlorination ${ }^{9,10}$ at $\mathrm{C}-3$ using $\mathrm{SO}_{2} \mathrm{Cl}_{2}$ (Scheme 1).

Two silanes, namely, crotylsilane and cinnamylsilane have been used for this study. It was envisaged that use of cinnamylsilane may lead to the formation of 3-(3'-phenylallyl) $\beta$-lactams very similar to biologically active ezetimibe (Zeita). Similarly, the use of crotyl silane could provide 3-(3'-methylallyl) $\beta$ lactams, another type of densely functionalized $\beta$ lactams. The studies were initiated with preparation of substituted silanes which were prepared by coupling reactions of Grignard reagent of the corresponding halide with chlorotrimethylsilane according to the procedure reported in literature. ${ }^{14}$

The reaction of crotyl magnesium chloride with chlorotrimethylsilane resulted in the formation of mixture of trans- and cis-crotyltrimethylsilanes and trimethyl- $\alpha$-methylallylsilanes in the ratio 80:20. However, the reaction of cinnamyl chloride with magnesium and trimethylsilylchloride resulted in the formation of only trans-cinnamyltrimethylsilane with a trace of cis-isomer. These silanes were purified by distillation. However, these silanes could not be fractionated and were used for further reaction as such. The structures of these silanes were confirmed by comparison of ${ }^{1} \mathrm{H}-\mathrm{NMR}$ data of products with the reported data in literature (Schemes 2 and 3). ${ }^{14}$

\subsection{Studies using crotylsilanes}

Initial studies were carried out using crotyl silanes as the nucleophile. Treatment of trans-3-chloro-3phenylthio- $\beta$-lactam 2a with crotyl silanes in the presence of $\mathrm{TiCl}_{4}$ at $0^{\circ} \mathrm{C}$ in $\mathrm{CH}_{2} \mathrm{Cl}_{2}{ }^{11}$ was monitored by
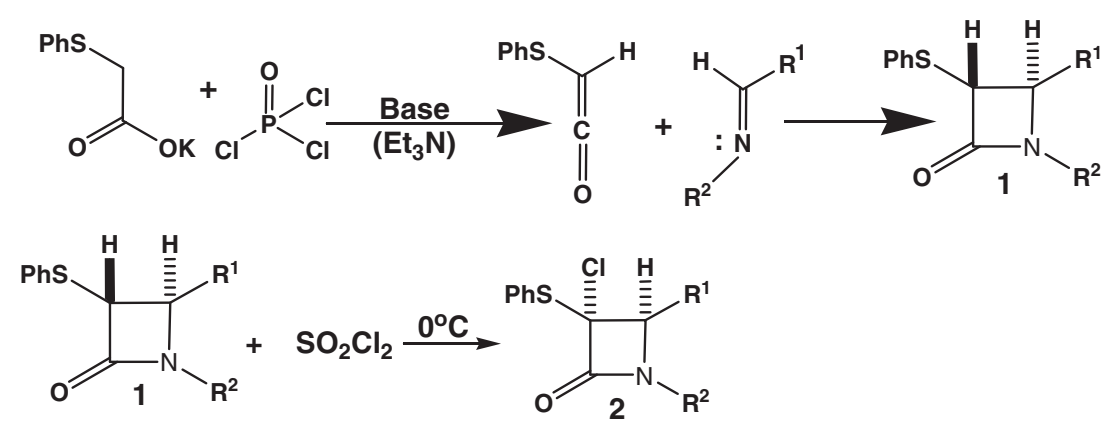

Scheme 1. Synthesis of starting compounds-3-phenylthioazetidin-2-ones 1 \& 3-chloro-3-phenylthioazetidin-2-ones 2. 


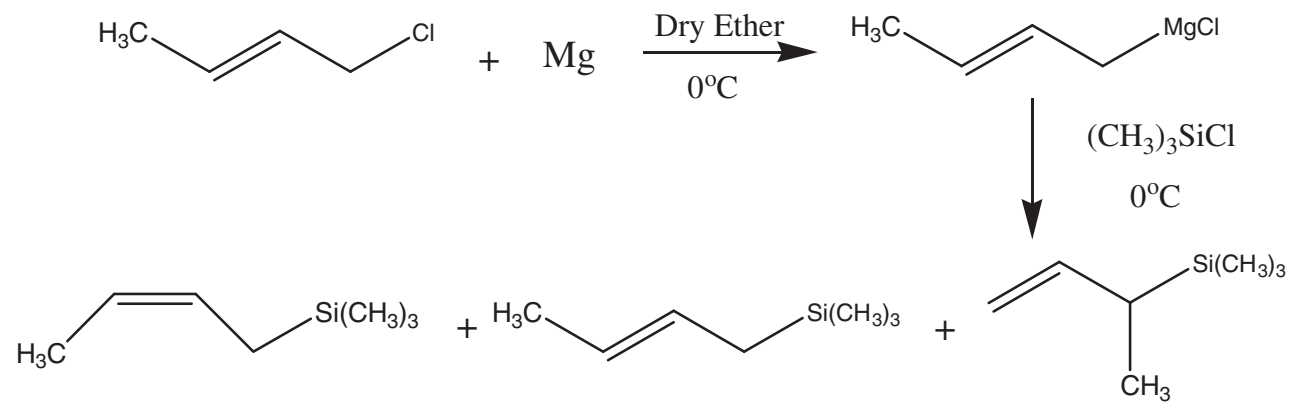

Scheme 2. Synthesis of crotyl silanes.

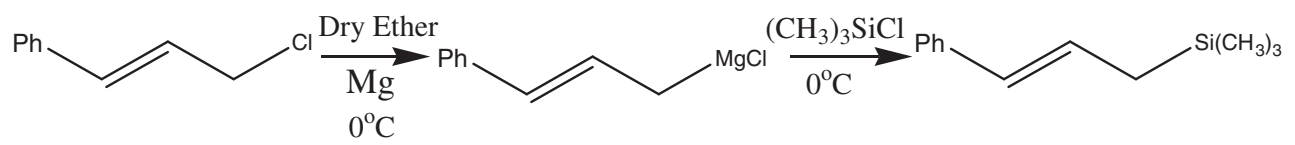

Scheme 3. Synthesis of cinnamyl silanes.

Table 1. Preparation of 3-(1'-methylallyl)-3-phenylthioazetidin-2-ones 3a-d.

\begin{tabular}{lccccc}
\hline Entry & $\mathbf{3}$ & $\mathbf{R}^{\mathbf{1}}$ & $\mathbf{R}^{\mathbf{2}}$ & Diastereomeric ratio & Yield $^{\mathrm{a}}(\%)$ \\
\hline 1 & $\mathbf{a}$ & $\mathrm{C}_{6} \mathrm{H}_{5}$ & $\mathrm{C}_{6} \mathrm{H}_{4}(\mathrm{OMe})(4)$ & $1: 1$ & 96.15 \\
2 & $\mathbf{b}$ & $\mathrm{C}_{6} \mathrm{H}_{4} \mathrm{Cl}(4)$ & $\mathrm{C}_{6} \mathrm{H}_{4}(\mathrm{Me})(4)$ & $1.33: 1$ & 64.5 \\
3 & $\mathbf{c}$ & $\mathrm{C}_{6} \mathrm{H}_{5}$ & $\mathrm{C}_{6} \mathrm{H}_{4} \mathrm{Br}(4)$ & $1.14: 1$ & 80.6 \\
4 & $\mathbf{d}$ & $\mathrm{C}_{6} \mathrm{H}_{5}$ & $\mathrm{C}_{6} \mathrm{H}_{5}$ & $1.23: 1$ & 85 \\
\hline
\end{tabular}

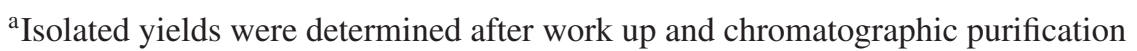

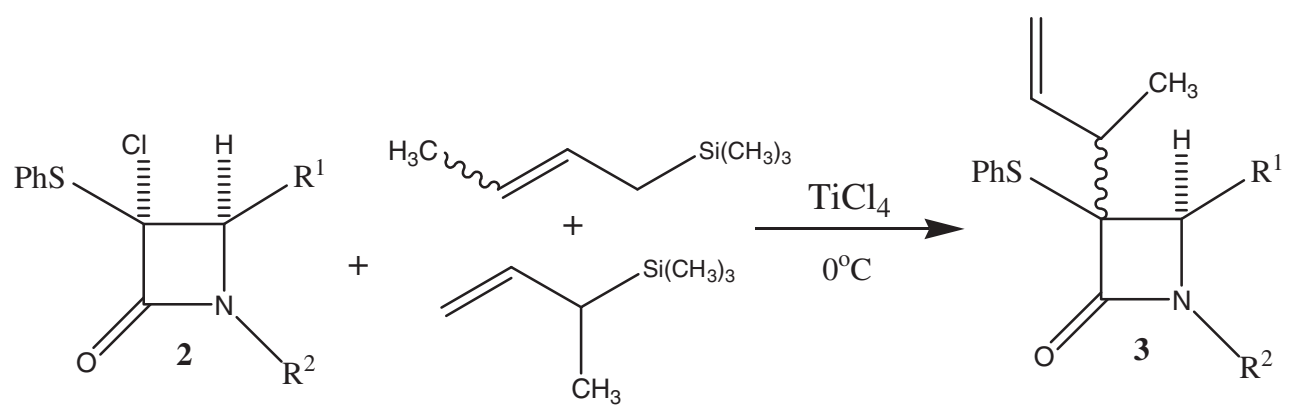

Scheme 4. Synthesis of 3- $\alpha$-methylallyl- $\beta$-lactams 3 .

TLC. The reaction mixture showed the appearance of single spot having $\mathrm{R}_{\mathrm{f}}$ slightly higher than that of the substrate. The ${ }^{1} \mathrm{H}$ NMR of the crude product indicated the formation of 3-(1'-methylallyl) $\beta$-lactams 3 instead of the expected 3-(3'-methylallyl) $\beta$-lactams. This upon purification and complete structural analysis confirmed the formation of compound $\mathbf{3}$ as mixture of two diastereomers. The diasteromeric ratio was determined by comparing peak area/integral values of multiplet of $\mathrm{C}^{\prime} 2-\mathrm{H}$ of attached allyl group which resonated at different chemical shifts $\delta$ 6.24-6.39 (minor isomer), 5.95-6.05 (major isomer). But these isomeric diastereomers could not be separated by column chromatography, since both isomers showed a single spot and same $R_{f}$ in TLC. The comparison of ${ }^{1} H$ NMR data of this compound with that of cis-3-allyl-3phenylthio- $\beta$-lactam, ${ }^{11}$ whose stereochemistry has been assigned based on X-ray crystallographic data, hinted the same stereochemical placement of allyl group at C-3. However, as this compound itself could not be crystallized, so no concrete proof of its stereochemistry can be given. From the types of product obtained, it can be inferred that in this reaction, out of two isomeric silanes, only the major one i.e., crotyl silane was found to be reacting. The reaction was found to be general and results are summarized in Table 1 (Scheme 4). 


\subsection{Studies using cinnamylsilanes}

Next, the reaction of $\mathbf{2}$ with cinnamylsilane under similar conditions was studied. This reaction also resulted in formation of the product arising from allylic shift, namely, 3-(1'-phenylallyl)-3-phenylthio- $\beta$ lactams. The product of this reaction, 4 was also found to be a mixture of two inseparable diastereomers, as detected by ${ }^{1} \mathrm{H}$ NMR spectrum of the crude product. However, the diastereomeric ratio was obtained from integration of protons of the substituent attached to the benzene ring as all other protons of the two diastereomers resonated at same value of chemical shift. The stereochemical placement of allyl group could not be assigned based on this data alone (Scheme 5, Table 2).

The transformation of trans-3-chloro-3-phenylthio$\beta$-lactam 2 into 3-(1'-methyl/phenylallyl)-3-phenylthio- $\beta$-lactams seems to proceed via intermediate formed by addition of C-3 carbocation to double bond facilitating the cleavage of $\mathrm{C}$-Si bond for formation of vinyl bond in the product as shown below (Scheme 6).

\subsection{Desulphurization studies}

Further, to prove the synthetic potential of this reaction and versatility of the products, all C-3-substituted- $\beta$-lactams

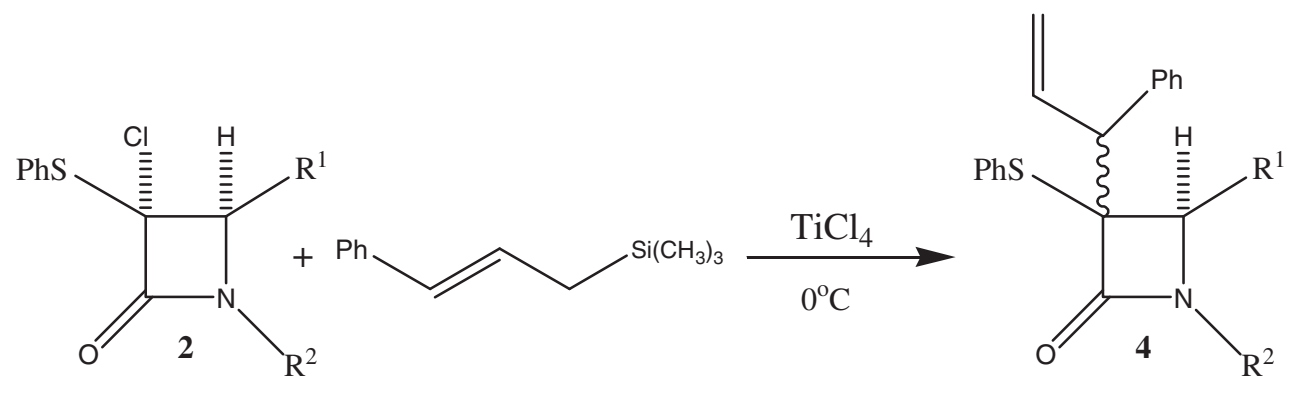

Scheme 5. Synthesis of 3- $\alpha$-phenylallyl- $\beta$-lactams 4.

Table 2. 3-(1'-phenylallyl)-3-phenylthioazetidin-2-ones, $\mathbf{4 a - d}$.

\begin{tabular}{cccccc}
\hline Entry & $\mathbf{4}$ & $\mathbf{R}^{\mathbf{1}}$ & $\mathbf{R}^{\mathbf{2}}$ & Diastereomeric ratio & Yield $^{\mathrm{a}}(\%)$ \\
\hline 1 & $\mathbf{a}$ & $\mathrm{C}_{6} \mathrm{H}_{5}$ & $\mathrm{C}_{6} \mathrm{H}_{4}(\mathrm{OMe})(4)$ & $1.6: 1$ & 62 \\
2 & $\mathbf{b}$ & $\mathrm{C}_{6} \mathrm{H}_{4} \mathrm{Cl}(4)$ & $\mathrm{C}_{6} \mathrm{H}_{4}(\mathrm{Me})(4)$ & $1.5: 1$ & 71 \\
3 & $\mathbf{c}$ & $\mathrm{C}_{6} \mathrm{H}_{5}$ & $\mathrm{C}_{6} \mathrm{H}_{4} \mathrm{Br}(4)$ & - & 71 \\
4 & $\mathbf{d}$ & $\mathrm{C}_{6} \mathrm{H}_{5}$ & $\mathrm{C}_{6} \mathrm{H}_{5}$ & - & 70 \\
\hline
\end{tabular}

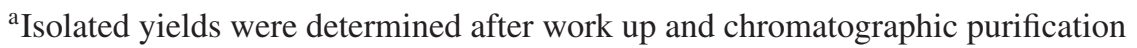

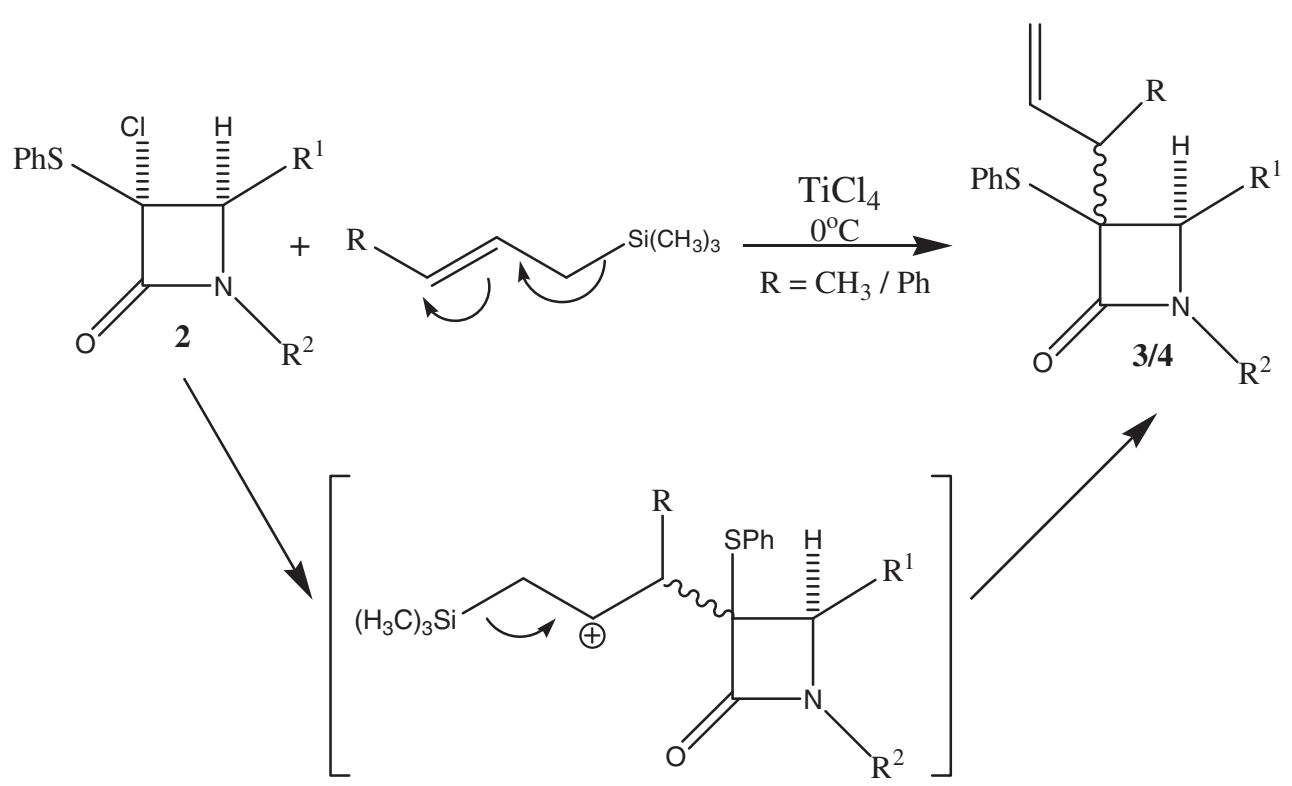

Scheme 6. Plausible reaction pathway for transformation of substrate 2 to product 3/4. 


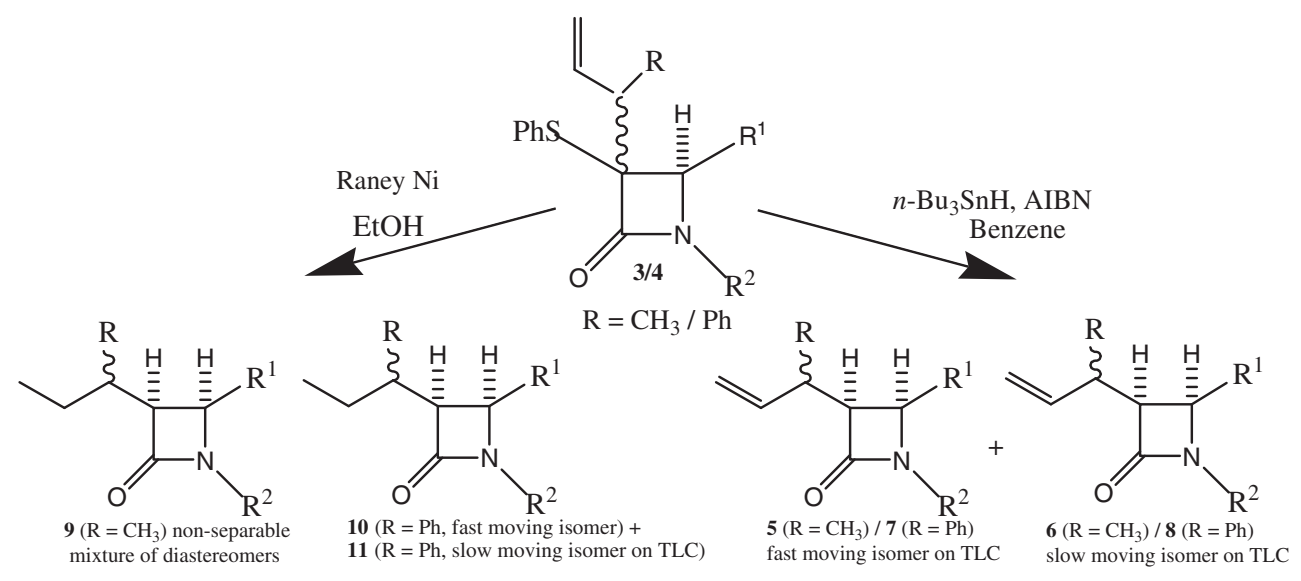

Scheme 7. Desulphurisation reactions of substrate 3 and $\mathbf{4}$.

Table 3. Reduction of $\mathbf{3}$ and $\mathbf{4}$ using $n-\mathrm{Bu}_{3} \mathrm{SnH}$.

\begin{tabular}{lccc}
\hline Entry & Substrate & Product & Yield $^{\mathrm{a}}(\%)$ \\
\hline 1 & 3a & $\mathbf{5 a}, \mathbf{6 a}$ & 56,24 \\
2 & $\mathbf{3 d}$ & $\mathbf{5 d}, \mathbf{6 d}$ & 55,27 \\
3 & $\mathbf{4 a}$ & $\mathbf{7 a}, \mathbf{8 a}$ & 49,25 \\
4 & $\mathbf{4 d}$ & $\mathbf{7 d}, \mathbf{8 d}$ & 44,29 \\
\hline
\end{tabular}

${ }^{a}$ Isolated yields were determined after work up and chromatographic purification.

Table 4. Reduction of $\mathbf{3}$ and $\mathbf{4}$ using Raney Ni.

\begin{tabular}{lccc}
\hline Entry & Substrate & Product & Yield $^{\mathrm{a}}(\%)$ \\
\hline 1 & $\mathbf{3 a}$ & $\mathbf{9 a}$ & 100 \\
2 & $\mathbf{3 d}$ & $\mathbf{9 d}$ & 80 \\
3 & $\mathbf{4 a}$ & $\mathbf{1 0 a}, \mathbf{1 1 a}$ & 33,46 \\
4 & $\mathbf{4 d}$ & $\mathbf{1 0 d}, \mathbf{1 1 d}$ & 31,64 \\
\hline
\end{tabular}

a Isolated yields are determined after work up and chromatographic purification.

were subjected to desulphurization reaction. Reduction of 3 using $n-\mathrm{Bu}_{3} \mathrm{SnH}$ ( 1 equiv.) in benzene catalysed by Azobisisobutyronitrile (AIBN) gave reduced product cis-3- $\alpha$-methylallyl- $\beta$-lactams 5 and 6. Interestingly, both the isomers of this reduced product were easily separable by column chromatography. The reduction reaction of $\mathbf{4}$ using the same reagent gave separable isomers of the reduced products, cis-3- $\alpha$-phenylallyl$\beta$-lactams 7 and $\mathbf{8}$. The stereochemistry at C-3 of these products was confirmed by the coupling constant between $\mathrm{C}_{3}-\mathrm{H}$ and $\mathrm{C}_{4}-\mathrm{H}$. However, the relative stereochemistry of the $\alpha$-group of the allyl substituent to these isomers could not be assigned on the basis of spectroscopic data alone. So, the two isomers were named as 'slow moving' and 'fast moving' diastereomers based on their $\mathrm{R}_{\mathrm{f}}$ values in TLC profile (Scheme 7, Table 3).

Reduction using Raney Nickel resulted in desulphurisation accompanied by hydrogenation of the double bond. Treatment of cis-3- $\alpha$-methylallyl- $\beta$-lactams, 3 with Raney Nickel gave cis-3- $\alpha$-methylpropyl- $\beta$ lactams 9, whose two diastereomers were inseparable on TLC and column chromatography. But reaction of $\mathbf{4}$ with Raney Nickel gave two separable diastereomers of the product cis-3- $\alpha$-phenylpropyl- $\beta$-lactam, 10 and $\mathbf{1 1}$ (Table 4).

\section{Conclusions}

In summary, this research work provides a facile and stereoselective method for the synthesis of cis-3-(1'methyl/phenylallyl)- $\beta$-lactams. Although these reactions could not give the desired products, they have resulted in the synthesis of new 3 -substituted-allyl $\beta$-lactams. Further, reduction using different reagents provides an easy access to two diastereomers of the products, which as such were unseparable. The synthetic potential of these compounds is under research in our lab.

\section{Supplementary Information (SI)}

All additional information pertaining to characterization of the representative compounds $(\mathbf{3 a}, \mathbf{4 a}, \mathbf{5 a}, \mathbf{6 a}$, 7a, 8a, 9a, 10a and 11a) using ${ }^{1} \mathrm{H}$ NMR and ${ }^{13} \mathrm{C}$ NMR techniques (Figures S1-S8) are given in the supporting information. Supplementary Information is available at www.ias.ac.in/chemsci.

\section{Acknowledgements}

We gratefully acknowledge the financial support for this work from TEQIP-II, UIET, P.U. Chandigarh, India.

\section{References}

1. Ojima I 1993 In The Organic Chemistry of $\beta$-Lactams G I George (Ed.) (New York: VCH) p.197 
2. Mascaretti O A, Boschetti C E, Danelon G O, Mata E G and Roveri O A 1995 Current Med. Chem. 1441

3. Hatanaka N, Abe R and Ojima I 1981 Chem. Lett. 101297

4. O'Boyle N, Carr M, Greene L, Bergin O, Nathwani S, McCabe T, Lloyd D, Zisterer D and Meegan M 2010 J. Med. Chem. $\mathbf{5 3} 8569$

5. Sandauayaka V P, Prashad A S, Yang Y, Williamson R T, Lin Y I and Mausour T S 2003 J. Med. Chem. 46 2569

6. (a) Manhas M S, Banik B K, Mathur A, Vincent J E and Bose A K 2000 Tetrahedron 56 5587; (b) Bose A K, Mathur C, Wagle D R and Manhas M S 2000 Tetrahedron 56 5603; (c) Ojima I 1995 Acc. Chem. Res. 28 383; (d) Banik B K, Manhas M S and Bose A K 1995 J. Org. Chem. 594714

7. Hanessian S and Alpegiani M 1989 Tetrahedron 45941

8. Tsuji J 1984 Synthesis 369

9. Jayaraman M, Manhas M S and Bose A K 1997 Tetrahedron Lett. 38709

10. Paquette L A, Rothhaar R R, Isaac M, Rogers L M and Rogers R D 1998 J. Org. Chem. 635463

11. Bari S S, Venugopalan P and Arora R 2003 Tetrahedron Lett. 44895

12. Arora R, Venugopalan P and Bari S S 2010 J. Chem. Sci. 122125

13. Reshma Arora R, Hundal G, Bhalla A and Bari S S 2015 J. Chem. Sci. 1271957

14. Slutsky J and Kwart H 1973 J. Am. Chem. Soc. 958678 